\title{
Evaluation of Cardiospermum halicacabum leaf compounds against human DihydroOrotate Dehydrogenase: a target for Rheumatoid Arthritis using Structure based Drug Designing
}

\author{
Priya Swaminathan, Lilly Saleena* \\ Department of Biotechnology, SRM University, Chennai, India.
}

\begin{tabular}{|c|c|}
\hline ARTICLE INFO & ABSTRACT \\
\hline Article history: & \multirow{12}{*}{$\begin{array}{l}\text { Rheumatoid arthritis [RA] is a disorder that makes the abnormal immune cells to assail the joints in the body } \\
\text { leading to pannus formation and destruction of the synovium. Human DiHydroOrotate DeHydrogenase } \\
\text { [hDHODH] inhibition is effective in controlling the proliferation of the abnormal immune cells. Though } \\
\text { technology has benefitted science and medicine in many ways, certain indigenous cures are still considered a } \\
\text { boom for various diseases. C. halicacabum an Indian medicinal plant is widely used as a home remedy for } \\
\text { arthritis pain. The novelty of this study relies on the extensive validations performed to obtain robustness of the } \\
\text { results. An E-pharmacophore model based on A771726- an experimentally resolved inhibitor of hDHODH was } \\
\text { screened against the phytocompounds in the leaf of } C \text {. halicacabum. These compounds were docked and further } \\
\text { validated statistical and ranking methods. Further, flexible docking was performed to understand the optimal } \\
\text { pose of the docked structures of Apigenin 7O-glucornide and Luteolin } 7 \text { O-glucornide. These optimal poses were } \\
\text { then simulated for } 10 \text { ns in a SPC environment which gave a very low RMSD value of } 1.5 \AA \text { A. These results were } \\
\text { comparable with the known inhibitor of hDHODH. Thus this study helps to understand and evaluate the } \\
\text { different probable inhibitors of hDHODH from C.halicacabum and suggests a mode of action for } \\
\text { phytochemicals of } C \text {. halicacabum against RA. }\end{array}$} \\
\hline Received on: $12 / 04 / 2017$ & \\
\hline Accepted on: 03/06/2017 & \\
\hline Available online: $30 / 08 / 2017$ & \\
\hline Key words: & \\
\hline Rheumatoid arthritis, $C$. & \\
\hline halicacabum, Dihydroorotate & \\
\hline Dehydrogenase, Screening & \\
\hline & \\
\hline & \\
\hline & \\
\hline & \\
\hline
\end{tabular}

\section{INTRODUCTION}

Human DiHydroOrotate DeHydrogenase [hDHODH] is a well studied target for Rheumatoid arthritis [RA] and various other proliferative diseases like multiple sclerosis, cancer and parasitic infections (Munier-Lehmann et al., 2013). It plays a role in denovo pyrimidine synthesis for faster proliferation of $\mathrm{T}$ cells. The proliferation of $\mathrm{T}$ cells is limited when hDHODH is inhibited. Thus the strategy of controlling proliferation by inhibiting hDHODH leads to abatement of symptoms hence leading to decline in the destruction caused by hyperactive immune cells (Fox et al., 1999). Leflunomide is a prodrug known

* Corresponding Author

Email: saleena.m@ktr.srmuniv.ac.in to inhibit hDHODH. The drug is effective with an IC50 value of $600 \mathrm{~nm}$ square but prolonged usage generally causes hepatic disorders that are classified as DILI (Drug Induced Liver Injury) (DeLeve and Kaplowitz, 2013). Thus the search for various alternatives that can be effective inhibitors as well as non toxic has been carried out in a number of studies like the fragment guided selection of hydroxycoumarins, anthranilic acids and fenamic acids based on the types of aminoacids present in the inhibitor active site (Fritzson et al., 2010).Another paper, describes Ascofuranone and Ascochlorin metabolites from a fungal strain show moderately good inhibitory action against hDHODH (Qi et al., 2009). Preceding this work, we have used a database of 1000 natural compounds to evaluate good inhibitors of hDHODH using novel E-pharmacophore validation techniques (Swaminathan et al., 2014). Here, our study deals with validating small molecules from traditionally used medicinal plant like Cadiospermum halicacabum as good inhibitors of hDHODH using new validation techniques. 
The validation is a multi course process where multiple statistical and repetitive tests are done to evaluate the confidence of the results obtained. The reproducible results are further crossdocked to evaluate the ligand using flexible hDHODH receptors from RCSB PDB. The optimal poses of the lead-like compounds were shortlisted. These poses are taken as the base structure for molecular dynamics to understand the behavior of the suggested lead-like phytocompounds against hDHODH in a simulated soluble environment.

The phytomolecules used in the study are the active ingredients of Indian medicinal plant Cardiospermum halicacabum. C. halicacabum has been used by many traditional medicinal systems as treatment of RA (Ganesan et al., 2011). Pharmacognasy properties of $C$. halicacabum as a crude drug have been studied and reported earlier (Zalke et al, 2013). In vitro studies have shown ethanolic crude extract of the leaf of $C$. halicacabum inhibit nitric oxide synthase and TNF- $\alpha$ (Sheeba and Asha, 2009).

Other studies have been used to prove its antiinflammatory nature (Babu and Krishnakumari, 2005; Sadique et al., 1987). Pharmacological validation of this plant revealed that the plant leaf compounds are safe and are not toxic up to $40 \mathrm{~g} / \mathrm{kg}$ in murine models (Pillai and Santhakumari, 1981). In other studies, the plant has been evaluated for its antimicrobial characteristics (Jeyadevi et al., 2012; Raza, 2013) as well as vectoricidal effects for mosquito borne diseases (Govindarajan and Sivakumar, 2012). Computational biology is a way to evaluate a probable inhibitor in a faster and definite manner in a workstation before experimentally determining the same (Marrone et al., 1997; Jorgensen, 2004). Virtual screening and molecular docking validation is considered to be a good way to evaluate the significance of the result. The commonly used method for validation is ROC curve (Vyas, 2008).Reproducing the same results in the experimental laboratory seems to be the biggest challenge in an in silico study (Kirchmair et al., 2015). Thus, this study helps to examine the results with respect to different aspects such as ranking, robustness, significance and pose difference to come forward with the best poses of the top hits of compounds from C. halicacabum.

This study would provide a comprehensive base for in depth analysis and search for alternative compounds that inhibit $\mathrm{hDHODH}$ and to explore an alternative mechanism of action for C. halicacabum inhibitors against $\mathrm{hDHODH}$. The study provides a summative proof of concept for the use of C. halicacabum as a treatment for RA.

\section{MATERIALS AND METHODS}

\section{Collection of SDF formats of Phytomolecules of C. halicacabum through Literature}

The compounds to be interrogated for their mechanism in Rheumatoid arthritis against the target human DHODH was collected through literature search (Veeramani et al., 2012; Jeyadevi et al., 2012).

\section{Protein Preparation}

The protein preparation wizard workflow of Schrodinger $9.2 \mathrm{v}$ for the $3 \mathrm{D}$-structures of proteins was implemented by adding hydrogens, assigning missing atoms and bond types, removing water molecules, and a refinement by completing missing side chains. The protein then was energy minimized using OPLS force field (Madhavi Sastry et al., 2013).

\section{E-Pharmacophore Screening}

Using the Advanced Search Tab in Schrodinger Maestro Suite, the E-pharmacophore was imported against the 19 compounds of Cardiospermum halicacabum. The screening resulted in finding matches that are similar to the ADHR features in the E-Pharmacophore.

\section{Ligand Preparation}

Ligands were prepared using Ligprep program of Maestro 9.2v (Madhavi Sastry et al., 2013). Each ligand was assigned an appropriate bond order and minimized using OPLS 2005 force field and ionized at $\mathrm{pH} 7$.

\section{Receptor Grid Generation}

The receptor grid file was generated using receptor grid generation option of Schrodinger $9.2 \mathrm{v}$ which represents physical properties of a volume of the receptor (specifically the active site) that are explored while performing docking (Madhavi Sastry et al., 2013). The grid is specified based on the ligand binding site in the co-crystal ligand.

\section{Validation Methods for Docking Programs}

Docking methods were evaluated using specificity, reproducibility using different programs and Receiver operating curves (ROC) plot. Docking using the decoy set of compounds that are mixed with the test compounds of a specific target. The ranking is used to plot receiver operating characteristic curves. ROC curves plot the sensitivity (Se) of a given docking / scoring combination against the specificity (Sp). ROC curve is a plot of true-positive versus false-positive rates for all compounds. The area under the ROC (AU-ROC) curve is the probability of active compounds being ranked earlier than decoy compounds. A ROC curve that passes through the upper left corner (100\% sensitivity and $100 \%$ specificity) has perfect discrimination (no overlap in the two distributions). Therefore, the closer the ROC curve is to the upper left corner, the higher the overall accuracy of the test (Triballeau et al., 2005).

\section{Molecular Docking Using Glide XP (Extra Precision)}

Molecular docking was performed for the prepared ligands using glide extra precision (XP) of Maestro 9.2v. This algorithm recognizes good hydrophobic, hydrogen-bonding, and metal-ligation interactions, and punishes steric clashes. Glide Score is based on ChemScore (Eldridge et al., 1997), but includes a steric-clash term, adds buried polar terms devised by 
Schrödinger to penalize electrostatic mismatches, and has modifications to other terms.

\section{Molecular Docking Using Ligand Fit}

The LigandFit docking procedure in Accelyrs discovery studio consists of: cavity detection for active site; and docking ligands to a selected site. Cavity detection, involves the use of a flood-filling algorithm. Its scoring function has been derived to predict binding affinities or to prioritize ligands relative to one another (Venkatachalam et al., 2003).

\section{Molecular Docking using iGEMDOCK}

iGEMDOCK v2.1 - A Graphical Environment for Recognizing Pharmacological Interactions and Virtual Screening[VS] is a docking tool for short listing pro-interactions like hydrogen bonds, electrostatic interactions and Van der Waals forces between the ligand and the receptor.

The energy value calculated by iGEMDOCK is the sum of binding energy, binding site pharmacophores energy and penalty of suboptimal ligand (Hsu et al., 2011).

BindingDB

It is a web-accessible database of experimental IC50 of protein considered to be drug-targets with small, drug-like molecules (Nicola et al., 2012). BindingDB contains 1,116,226 binding data, for 6,974 protein targets and 478,572 small molecules. It is expected that the enhanced access to data provided by this resource will facilitate drug-discovery and the development of predictive computer models of binding.

\section{Crossdocking}

To study the specificity of the receptors and ligands, when the receptors are all the same protein but in different conformations can yield valuable information about the effects of induced fit upon binding (Totrov and Abagyan, 2008). Glide Cross Docking (XGLIDE) automates cross docking calculations with Glide. The flexibility of the receptor is taken into account for ligand specificity. It is a practical method where experimentally derived structures are used to improve docking predictions

\section{Free Energy Calculation using Prime MM/GBSA}

Molecular mechanics with generalized Born and surface area solvation (MM/GBSA) is a popular approach performed using Prime option of Schroedinger's Maestro suite. MM/GBSA quantifies the difference in energy between the free and the complex state of both the ligand and the protein after energy minimization. The energies of the complex were calculated using OPLS 2005 (optimized potentials for liquid simulations force field) and generalized Born and surface area solvation model. This program is used to predict the strain energy and free energy of binding for each ligand (Du et al., 2011).

\section{Dynamics Simulation}

A solvated system was subjected to energy minimization using steepest descent algorithm. MD simulations on Desmond first relax and minimize the receptor alone or the complex. Further MD simulations were carried out for desired period of time at a constant temperature of $300 \mathrm{~K}$ and constant pressure of $1 \mathrm{~atm}$ with a time step of $2 \mathrm{fs}$ by means of Nose-Hoover thermostat method. Molecular dynamics is a complement experiment to explain a molecular mechanism as well as describe a system at equilibrium (Karplus and McCammon, 2002). The energy and trajectory atomic coordinates for all simulations were recorded at every 1.2 ps and $4.8 \mathrm{ps}$.

\section{RESULTS AND DISCUSSION}

\section{Phytochemicals of C. halicacabum}

Cardiospermum halicacabum is an uncultivated food crop, widely distributed in India and consumed as green leafy vegetable. The anti-inflammatory activity of ethanol extract of $C$. halicacabum leaves against carrageenan-induced rat paw edema has already been observed experimentally in rats (Huang et al., 2011). The ethanol extract of the $C$. halicacabum suppresses the production of TNF-alpha and nitric oxide in human peripheral blood mononuclear cells (Venkatesh and Krishnakumari, 2009). Jeyadevi et al, (2012) worked on the ethanolic extract for its chemical constituents using GC-MS and FT-IR. This yielded in peaks for flavonoids and phenolic acids. Further literature searching resulted in some other compounds like cardiospermin, Calycosin, Rutin, Protocatechaldehyde, Quebrachitol, Phloridzin, Prunin and Ferulic acid (Veeramani et al., 2012; Jeyadevi et al., 2012). In total 19 compounds were shortlisted from the ethanolic leaf extract of C. halicacabum. Most of the compounds were flavanoids or alkaloids.

\section{E-Pharmacophore Screening}

A validated E-Pharmacophore for $\mathrm{hDHODH}$ published in a previously done work (Swaminathan et al., 2014) was used to screen the 19 compounds. E- Pharmacophore or structure based pharmacophore is a scaffold of important features and their distances that specify the efficiency of an inhibitor. The validated E-Pharmacophore had 4 features viz. an Acceptor, a Donor, a hydrophobic group and a Ring structure. All these features correspond to the hydrogen bonds and hydrophobics interactions obtained in the X-ray Crystallographic Structure of hDHODH (1D3H) (Swaminathan et al., 2014). The essential features represented in the E-pharmacophore ADHR at specific distances was screened against the 19 compounds to identify hits that have inhibitor like features. Fitness score measures the degree of equivalence between the ligand conformer and the hypothesis (Sotriffer, 2011). The E-pharmacophore which was based on A771726 - a known potent inhibitor of hDHODH was screened against the 19 compounds of $C$. halicacabum to sought out structurally similar compounds in the leaf that have similar features like the inhibitor. The E-pharmacophore with essential hDHODH inhibitory features was further taken for database screening to identify the novel molecules. Table 1 lists the 8 compounds that showed fitness score equal or greater than 1.The 
highest score obtained was for coniferaldehyde with 1.78 while 7 other compounds were equal to or higher than 1.3 fitness score.

Table 1: Fitness Scores of compounds screened with E-Pharmacophore.

\begin{tabular}{cc}
\hline Compounds & Fitness score \\
\hline Coniferaldehyde & 1.78 \\
Quercetin & 1.71 \\
Chryseriol & 1.67 \\
Prunin & 1.65 \\
Apigenin & 1.62 \\
Calycosin & 1.53 \\
Caftaric acid & 1.52 \\
Luteolin7O- Glucornide & 1.32 \\
\hline
\end{tabular}

Table 1 suggests that coniferaldehyde and quercetin along with Chryseriol have similar features at distances matching with that of the pharmacophore hypothesis. All the hits were superimposed on the E-pharmacophore to manually inspect the degree of similarity (Figure 1). Thus compounds that are top hits in pharmacophore screening may dock with higher score. It is also accomplished that all of the 8 compounds obtained have features that match at various degrees to the known inhibitor of human DHODH.

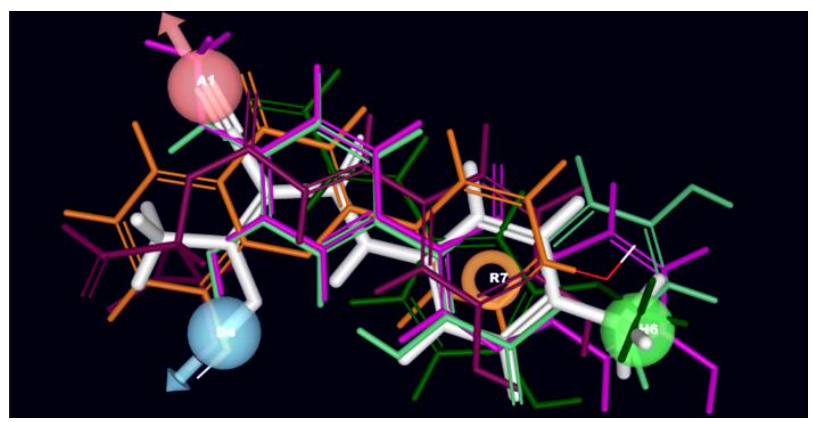

Fig. 1: Superimposition of the different hits after screening with the Epharmacophore (White), Coniferaldehyde(green), Quercetin(cyan), apigenin (orange), calycosin(magenta) and caftaric acid(purple).

\section{Glide Docking}

Although only fewer hits were screened in the previous step, all 19 phytocompounds were docked to identify the binding mode of the active phytoconstituents with the inhibitor active site of human DHODH receptor. The glide XP G-score ranged from$11.28 \mathrm{Kcal} / \mathrm{mol}$ to $-8.22 \mathrm{Kcal} / \mathrm{mol}$. The hit compounds which possess high glide score compared with the known inhibitor A771726 (Glide XP G-score $=-8.15 \mathrm{Kcal} / \mathrm{mol}$ ) was considered as good hits (Table 2).

Out of 19 compounds, only ten compounds showed XP G-score greater than A771726. The XP G-score is generated using scoring functions that consider the bonded and non-bonded interactions as well as penalties for rotatable bonds and polar group interactions in active site. These hit compounds were examined for their bonded and non bonded interactions with human DHODH. In an earlier study done to find the most effective inhibitor against human $\mathrm{DHODH}$, it was discussed that an ideal human DHODH inhibitor must possess Van der Waals, hydrophobic and negatively charged interactions with the receptor.
The binding mode of the identified hits was inferred by studying the essential bonded and non-bonded interactions. Our identified hits show good electrostatic (XP electro) and hydrophobic interactions (XP PhobEn) with the receptor DHODH. A comparison of docking poses for known and identified hits suggests that the hits show almost similar binding mode with the active site residues of DHODH (Pro52, Ala55, Thr63, Leu67, Arg136 and Tyr356).

Table 2: Docking results for the phytomolecules against human DHODH using GlideXP.

\begin{tabular}{|c|c|c|c|c|}
\hline S.No & $\begin{array}{l}\text { Compound } \\
\text { Name }\end{array}$ & $\begin{array}{c}\text { Glide XP } \\
\text { Score } \\
\text { (Kcal } / \mathrm{mol}) \\
\end{array}$ & $\begin{array}{c}\text { Glide } \\
\text { Energy } \\
\text { (Kcal/mol) } \\
\end{array}$ & Interactions \\
\hline 1. & Caftaric Acid & -11.28 & -45.16 & $\begin{array}{l}\text { Ser215, Asn284, } \\
\text { Asn212, } \\
\text { Lys100,Ser120 }\end{array}$ \\
\hline 2. & $\begin{array}{l}\text { Luteolin7O- } \\
\text { glucornide }\end{array}$ & -10.85 & -55.36 & Tyr356, Pro52 \\
\hline 3. & Phloridzin & -10.44 & -46.43 & Tyr 38 \\
\hline 4. & Prunin & -10.10 & -37.03 & Pro52 \\
\hline 5. & Chlorogenic acid & -9.84 & -50.02 & Pro52(2) \\
\hline 6. & Chryseriol & -9.18 & -39.47 & - \\
\hline 7. & Calycosin & -9.05 & -37.92 & Leu359 \\
\hline 8. & Apigenin & -8.69 & -37.61 & Ala55 \\
\hline 9. & $\begin{array}{l}\text { Apigenin7O- } \\
\text { glucornide }\end{array}$ & -8.22 & -57.87 & Pro52 \\
\hline 10. & A771726 & -8.15 & -41.02 & Tyr356,Arg136 \\
\hline
\end{tabular}

\section{Binding Mode of Caftaric Acid}

Caftaric acid, which was one of the hits in the Epharmacophore screening, had the highest Glide XP Gscore of $11.22 \mathrm{Kcal} / \mathrm{mol}$ among other hits. This compound revealed a network of hydrogen bonds with receptor residues like Ser215, Asn284, Asn212, Lys100 and Ser120. This compound donates a Hydrogen bond with side chain of Asn212(1.73 ̊́) and accepts 4 hydrogen bonds from the side chains of Ser215, Asn284, Lys 100

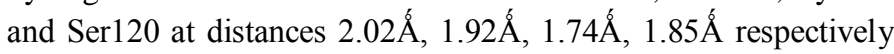
(Figure 2). However the binding mode is not in the same pocket as that of the known inhibitor site but farther away in a deeper pocket near substrate binding site of FMN. Thus, compared to A771726, caftaric acid binds in a region away from the known binding site and thus cannot be considered as a good hit among all the hits obtained as compared to the known inhibitor A771726. It has rich hydrophobic interactions with Tyr147, Val134, Tyr356 and Val143.

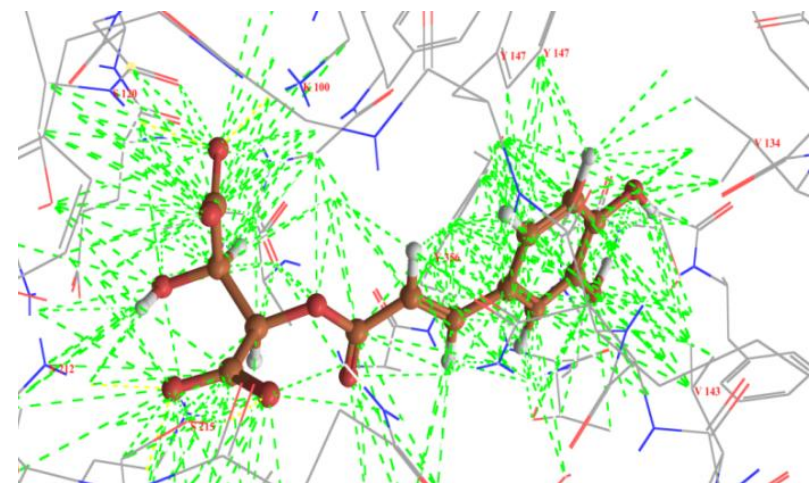

Fig. 2: Binding mode of Caftaric acid. 



Fig. 3: Binding modes of A. Luteolin 7O-glucornide B. Phloridzin C. Prunin (green) Chlorogenic acid (black) and Apigenin 7O-glucornide (blue) D. Chryseriol (pink), Calycosin (purple) and Apigenin (black)

\section{Binding Mode of Luteolin-7O Glucornide}

Rhamnazin, a flavonol, scores $-10.70 \mathrm{Kcal} / \mathrm{Mol}$ and donates a hydrogen bond to a main chain of Pro52 backbone at the carboxyl oxygen $(1.71 \AA$ ) $)$ and donates another hydrogen to the side chain of Tyr356(2.02 ̊́).Its XP vdW and XP PhobEn are -0.69 and -0.17 respectively. Its hydrophobic score is much better than the other molecules compared. It makes hydrophobic interactions with Met43, Leu46, Pro52, Ala55, Phe62, Phe98, Pro364, Tyr356 and Leu359. The binding mode is quiet similar to the binding mode of A771726 with Tyr356 (2.91 Á). The hydrophobic interactions of this molecule contribute to the high Gscore obtained and indicates a possible effective inhibitor activity of the compound against human DHODH (Figure 3A).

\section{Binding Mode of Phloridzin}

Phloridzin is a flavonol that binds with a Gscore of $10.44 \mathrm{Kcal} / \mathrm{mol}$ and forms a hydrogen bond with Tyr38 (1.88 Á).The side chain of Tyr38 accepts hydrogen atoms from the Hydroxyl group present in the dihydroxyphenyl ring of phloridzin. The electrostatic interaction contributes to increase in the XP Electro contribution to -0.25 . The XP Vdw contribution is about 43.07 with hydrophobic bonds formed with Tyr38, Leu42, Met43, Leu46,Pro52 Ala55, Ala59, Leu68, Val134, Leu359 and Pro364 .Tyr38 is in the same pocket as that of Tyr356 thus the binding mode is comparable with A771726 (Figure 3B).

\section{Binding Mode of Prunin, Chlorogenic Acid and Apigenin 70 - Glucornide}

Prunin, chlorogenic acid and apigenin 7Oglucornide all interact with the amino acid Pro52 forming bonds at an average distance of hydroxyl group at 2.1 ̊́. Chlorogenic acid forms two bonds with Pro52.The high XPGscore for prunn is accounted by the high $\mathrm{Vdw}$ interaction score of-32.96 Kcal $/ \mathrm{mol}$ which is comparable with that of A771726 having a score of -31.94 Kcal /mol. Apigenin 7oglucornide shows similar XP PhobEn of -2.82 with A771726 having XPPhoben score of -2.00. The Hydrophobic interactions with Met43, Leu46, Ala55, Ala59, Phe62 and Leu67 and Tyr356 and Leu364 were common for the three molecules compared (Figure 3C). Chlorogenic acid had high XP Phoben and $\mathrm{XP}$ vdw compared apigenin $7 \mathrm{O}$ glucornide but lower than Prunin.

\section{Binding Mode of Chryseriol, Calycosin and Apigenin}

Among the three, Chryseriol has the highest XP PhobEn contribution compared to the others. They had a glide XPGscore of $-9.18,-9.05$ and $-8.69 \mathrm{Kcal} / \mathrm{mol}$. the amino acid interactions forming Hydrogen bonds were with Ala55 and Leu359 (Figure 3D). The hydrophobic interactions were with the amino acids Met43, Leu46, Ala55, Ala59, Phe62 and Leu67 and Tyr356 and Leu364. The molecules are consecutive in the list of glide XP results and only differ majorly in their glide evdw score of -37.37 , -33.33 and -34.43 respectively. Based on the binding mode 
analysis it is clear that all the hits obtained are comparable to the known inhibitor and its significance is maintained by binding in an equivalent manner as the known inhibitor molecule. The exception in this is Caftaric acid which has a good glide score and energy but the Binding pocket is a little deeper than the inhibitor binding site. To evaluate if caftaric acid is a significant hit, docking experiment is validated by using three validation methods.

\section{Validation of the Docking Results}

The docking results significance can be validated by many methods. The validation aims to corroborate the robustness of the results with its reliability. The results will throw light on the factors that affect a docking experiment, like the docking algorithm, the use of large data set of decoys and performance of the docking tool with known and unknown active molecules.

\section{ROC Plot Analysis}

Receiver operator Characteristic [ROC] curve is done to justify the sensitivity and the specificity of the receptor towards the phytocompounds. Docking program was validated using ROC which describes the ability of docking method to avoid false positives and false negatives. The docking experiment is modified to accommodate the 19 compounds of $C$. halicacabum and 300 compounds from the Asinex decoy set in the ratio 1:15. The decoys have been used traditionally to evaluate the sensitivity of a pharmacophore while in other studies have been used for evaluating the specificity of a receptor. Enrichment was calculated based on how well the candidate or annotated compounds were retrieved from the decoy database. Here the ROC curve obtained had an Area under Curve (AUC) value of 0.72 which is significant according to literature survey (Figure 4). $79 \%$ of the actives compounds (19 compounds) were retrieved as hits. The top four hits of Glide XP were in correlation to the top four hits of the decoy set infused actives docking.

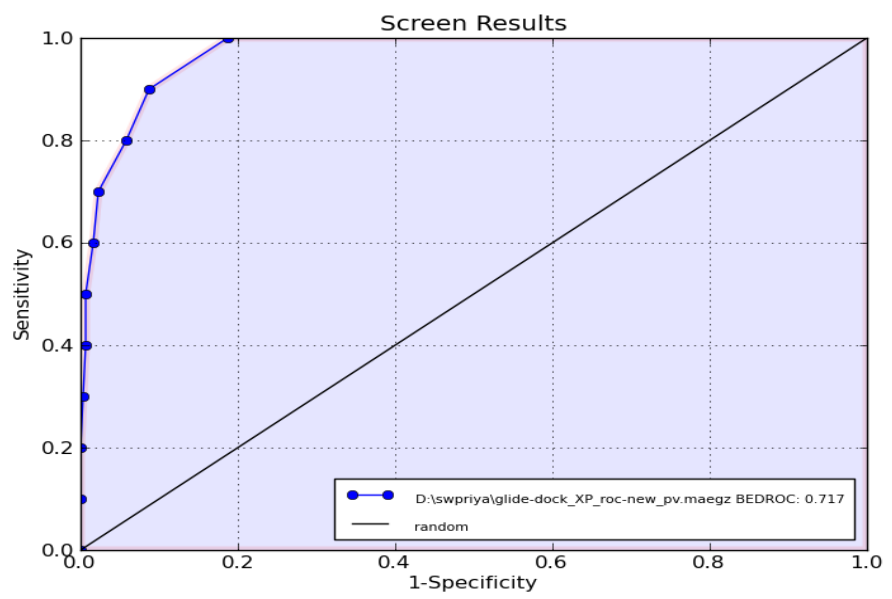

Fig. 4: Receiver Operator characteristic curve against decoys

\section{Docking with Several Different Algorithms}

The docking algorithm plays a humongous role in retrieving the true positives as results. Different algorithms were used to check the robustness of the result obtained. Two docking algorithms were used for docking the 19 phytocompounds of $C$. halicacabum with the receptor $1 \mathrm{D} 3 \mathrm{H}$.

\section{Docking In Accelrys Ligandfit Tool}

The docking algorithm of Ligandfit is different from GlideXP as it searches for shape complementarities of the ligand with the receptor rather than hierarchical filtering of ligand poses in the receptor pocket. This amounts to searching of ligand pose in all bumps or openings in the receptor structure. Ligandfit was able to define the receptor to have 3 binding pockets. When the 19 ligands along with A771726 were probed in 3 different pockets, good dockscore and PLP score were obtained for the different ligands only in the inhibitor binding site.

Table 3 shows 7 hits having better dockscore compared to A771726. The dockscore ranges from 73.01 to $55.78 \mathrm{Kcal} / \mathrm{mol}$.

Table 3: Docking results for the phytomolecules against human DHODH using Accelrys Ligandfit.

\begin{tabular}{ccccc}
\hline $\begin{array}{c}\text { Compound } \\
\text { Name }\end{array}$ & $\begin{array}{c}\text { Dockscore } \\
\text { Kcal/mol }\end{array}$ & $\begin{array}{c}\text { PLP1 } \\
\text { Kcal/mol }\end{array}$ & $\begin{array}{c}\text { PMF } \\
\text { Kcal/mol }\end{array}$ & Interactions \\
\hline $\begin{array}{c}\text { luteolin7O- } \\
\text { glucornide }\end{array}$ & 73.01 & -121.77 & -122.38 & $\begin{array}{c}\text { Arg136, Tyr38, } \\
\text { Pro52, Gln47 }\end{array}$ \\
$\begin{array}{c}\text { apigenin7O- } \\
\text { glucornide }\end{array}$ & 70.90 & -114.15 & -116.5 & Pro364, Tyr38 \\
prunin & 67.48 & -117.48 & -100.99 & Arg136 \\
chlorogenic acid & 65.06 & -99.3 & -80.57 & Arg136, Pro 52, \\
& & & & Gln47 \\
chryseriol & 63.01 & -102.1 & -72.55 & Arg136, \\
phloridzin & 59.35 & -104.28 & -88.9 & Thr63, Met43 \\
caftaric acid & 57.52 & -88.22 & -60.35 & Arg136 \\
A771726 & 55.78 & -102.7 & -67.4 & Tyr356, Arg136 \\
\hline
\end{tabular}

Luteolin 7O- glucornide was the top hit here compared to caftaric acid in Glide XP. The type of interactions made by Luteolin 70 glucornide is the similar with Pro52. The bond distance is comparable. All the other hits including caftaric acid bound in the same pocket as that of the inhibitor Leflunomide. Figure 5 gives the binding pose of the top hit.

The remaining two predicted binding sites gave "no poses docked". This means nil results were obtained.

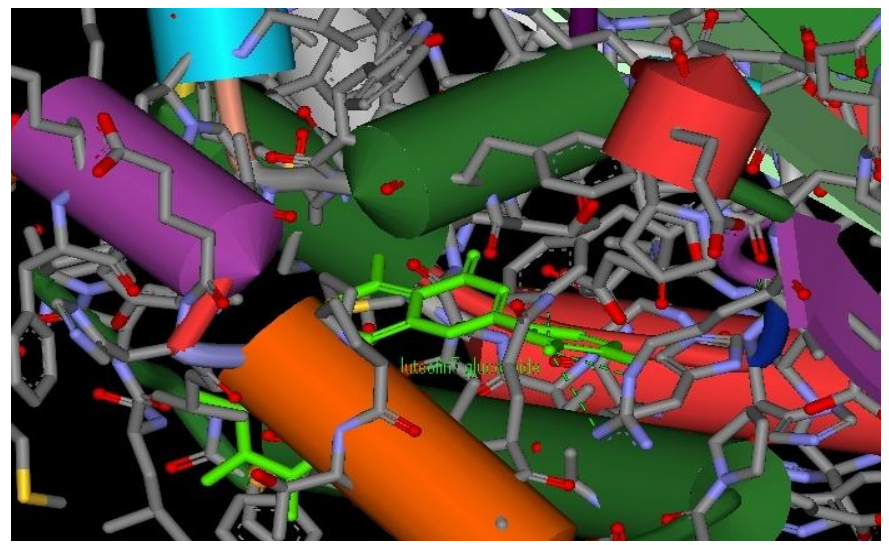

Fig. 5: Docked pose of Luteolin 7O-glucornide (light green) with hDHODH a predicted by Accelrys Ligand Fit tool. 


\section{Docking with the iGEMDOCK Tool}

iGEMDOCK tool uses evolutionary steps of genetic algorithm to find docked poses of ligands. The poses generated are survivors of goodness of fit score. The docking results of the 19 phytocompounds with the human DHODH receptor gave 6 hits with Luteolin 7O-glucornide as the best hit followed by apigenin 70 glucornide. The hits generated were all better energy value compared to the known inhibitor A771726. The evolutionary based algorithms tends to select those hits that are better among a population of hits or poses that have better energy than the earlier hits obtained. This iterative process only ceases when no better pose with better energy can be generated.

Table 4: Docking results for the phytomolecules against human DHODH using iGEMDOCK tool

\begin{tabular}{|c|c|c|c|c|}
\hline 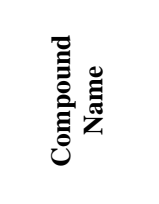 & 预总 & 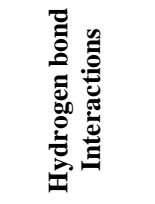 & 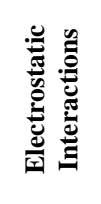 & 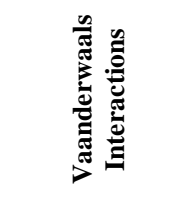 \\
\hline $\begin{array}{l}\text { Luteolin7O- } \\
\text { glucornide }\end{array}$ & -153.22 & $\begin{array}{c}\text { Arg136,Tyr } \\
\text { 38,Pro52, } \\
\text { Gln47, } \\
\text { His56,Thr6 } \\
\text { 3, Tyr356 } \\
\end{array}$ & - & $\begin{array}{c}\text { Ala59, Met43, } \\
\text { His56, Ala55, } \\
\text { Phe62, Phe98, } \\
\text { Leu359, } \\
\text { Thr360, Pro364 }\end{array}$ \\
\hline $\begin{array}{l}\text { Apigenin7O- } \\
\text { glucornide }\end{array}$ & -147.00 & $\begin{array}{c}\text { Arg136,Tyr } \\
\text { 38,Pro52,Gl } \\
\text { n47,His56, } \\
\text { Tyr356 }\end{array}$ & Arg136 & $\begin{array}{l}\text { Pro364, Tyr38, } \\
\text { Pro52, Ala55, } \\
\text { His56, Ala59, } \\
\text { Phe62, Phe98, } \\
\text { Leu359,Thr360 }\end{array}$ \\
\hline Phloridzin & -141.52 & $\begin{array}{l}\text { Arg136,Pro } \\
\text { 52, Gln47, } \\
\text { Ala59, } \\
\text { Leu68, }\end{array}$ & - & $\begin{array}{l}\text { Pro52, Ala55, } \\
\text { His56, Ala59, } \\
\text { Phe62, Arg136, } \\
\text { Tyr356,Leu359 } \\
\text { Thr360 Pro364 }\end{array}$ \\
\hline prunin & -141.10 & $\begin{array}{c}\text { Arg136,Thr } \\
63, \text { Tyr38 }\end{array}$ & - & $\begin{array}{c}\text { Ala59, Met43, } \\
\text { His56, Ala55, } \\
\text { Phe62, Phe98, } \\
\text { Leu359,Thr360 } \\
\text { Pro364, Tyr38, } \\
\text { Arg136 }\end{array}$ \\
\hline $\begin{array}{l}\text { chlorogenic } \\
\text { acid }\end{array}$ & -138.50 & $\begin{array}{c}\text { Arg136,Tyr } \\
\text { 147,Thr63, } \\
\text { His56, } \\
\text { Tyr356 }\end{array}$ & - & $\begin{array}{c}\text { Arg136,Tyr356 } \\
\text {,Pro52,Ala55, } \\
\text { His56,Phe98, } \\
\text { Val134,Leu359 } \\
\text {,Thr360 }\end{array}$ \\
\hline caftaric acid & -124.09 & $\begin{array}{c}\text { Gln47,Thr6 } \\
\text { 3,Arg136, } \\
\text { Tyr356, } \\
\text { Pro364 }\end{array}$ & Arg136 & $\begin{array}{c}\text { Gln47,Pro52, } \\
\text { Ala55,His56,Al } \\
\text { a59, Phe98, } \\
\text { Arg136,Tyr356 } \\
\text { Leu359,Thr360 }\end{array}$ \\
\hline A771726 & -123.8 & $\begin{array}{l}\text { Tyr356, } \\
\text { Arg136 }\end{array}$ & - & $\begin{array}{c}\text { Gln47,Pro52, } \\
\text { Ala55,His56,Al } \\
\text { a59,Phe98,Arg } \\
\text { 136,Tyr356, } \\
\text { Leu359,Thr360 } \\
\text {,Pro364 }\end{array}$ \\
\hline
\end{tabular}

Table 4 summarizes the iGEMDOCK result in such a way that it gives details of the hits in the format of Energy and the nonbonded interactions like hydrogen bonds, Van der Waals interactions and electrostatic interactions. The energy score ranged from -153.22 to $-123.8 \mathrm{Kcal} / \mathrm{mol}$. Luteolin $7 \mathrm{O}$-glucornide and
Apigenin 70- glucornide were the top two hits. All the top hits had similar interaction in the inhibitor site. Figure 6 depicts the binding pose for Luteolin 70-glucornide as predicted by iGEMDOCK. It shows Hydrogen bonds as dashed yellow lines with Arg136, Tyr38, Pro52, Gln47, His56 and Tyr356.

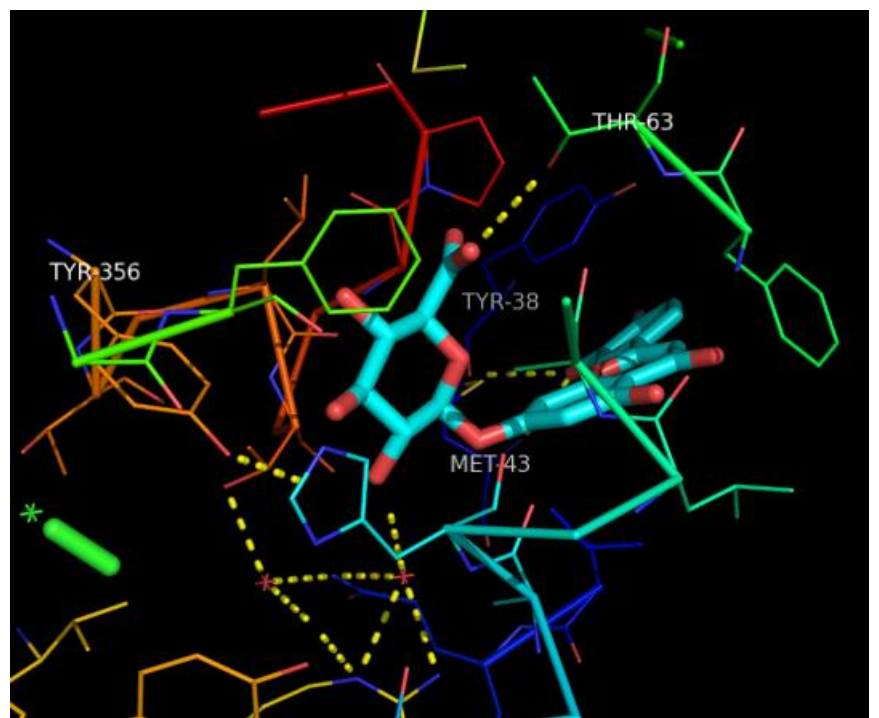

Fig. 6: Docked pose of top hit (luteolin7O-glucornide) as predicted by iGEMDOCK

\section{Validation using Curated BindingDB}

BindingDB is a database that contains binding affinity information of different receptors for different ligands in different organisms. The database was curated for small molecules that inhibit human DHODH specifically and which neither binds with other species DHODH receptor nor with other protein drug target receptors. Such potent, crosschecked and specific small molecules were collected and made into a phase database file as curated BindingDB for human DHODH. A total of 229 molecules were found that were very specific to human DHODH with high IC50 value. These inhibitors along with the nineteen phytomolecules were docked with human DHODH to figure out where the phytomolecules rank themselves compared to known, potent, specific inhibitors.333 poses were generated where caftaric acid and Luteolin 7O-glucornide and appeared among the top 25 of the hits. A771726 among other known inhibitors was ranked 155 with a XP GScore of $-8.32 \mathrm{Kcal} / \mathrm{mol}$. Caftaric acid and Luteolin $7 \mathrm{O}$ glucornide had XP Gscore of -11.28 and $-10.85 \mathrm{Kcal} / \mathrm{mol}$ which is significantly higher. However the binding pocket differed for caftaric acid in a deeper tunnel farther away from the inhibitor binding site .Other compounds which were ranked well than A771726 were phloridzin, prunin, chlorogenic acid, chryseriol, calycosin and apigenin. Their XP Gscore and binding site were analogous with A771726.

The Table 5 depicts the hits when sorted with glide energy. Table depicts the known compounds with its IC50 value while the $C$. halicacabum compounds do not have a known IC50 value against the target. 
Table 5: Glide XP score of Binding DB based known inhibitors against hDHODH.

\begin{tabular}{clcc}
\hline S. & \multicolumn{1}{c}{ TITLE } & $\begin{array}{c}\text { ENZYMOLOGIC } \\
\text { IC50 (nM) }\end{array}$ & $\begin{array}{c}\text { GLIDE } \\
\text { ENERGY } \\
\text { Kcal/mol }\end{array}$ \\
\hline 1 & apigenin7O- glucornide & - & -57.87 \\
2 & luteolin7O- glucornide & - & -55.36 \\
3 & CHEMBL370008 & 2000 & -51.65 \\
4 & CHEMBL2177115 & 1270 & -51.16 \\
5 & CHEMBL154336 & 370 & -50.71 \\
6 & US8536165 & 190 & -50.09 \\
7 & chlorogenic acid & - & -50.02 \\
8 & Phloridzin & - & -49.66 \\
9 & CHEMBL155548 & 290 & -49.43 \\
10 & CHEMBL1076869 & 3300 & -49.38 \\
11 & CHEMBL358435 & 8400 & -49.35 \\
12 & CHEMBL154908 & 2900 & -49.20 \\
13 & CHEMBL193365 & 11 & -49.11 \\
14 & CHEMBL1929439 & 27 & -48.95 \\
15 & Biphenyl-4-lcarbamoyl & 44 & -48.61 \\
& Thiophene Analog 5 & & -48.42 \\
16 & Biphenyl-4- & 1 & \\
& ylcarbamoyl Thiophene & & \\
\hline & Analog 8 & & \\
\hline
\end{tabular}
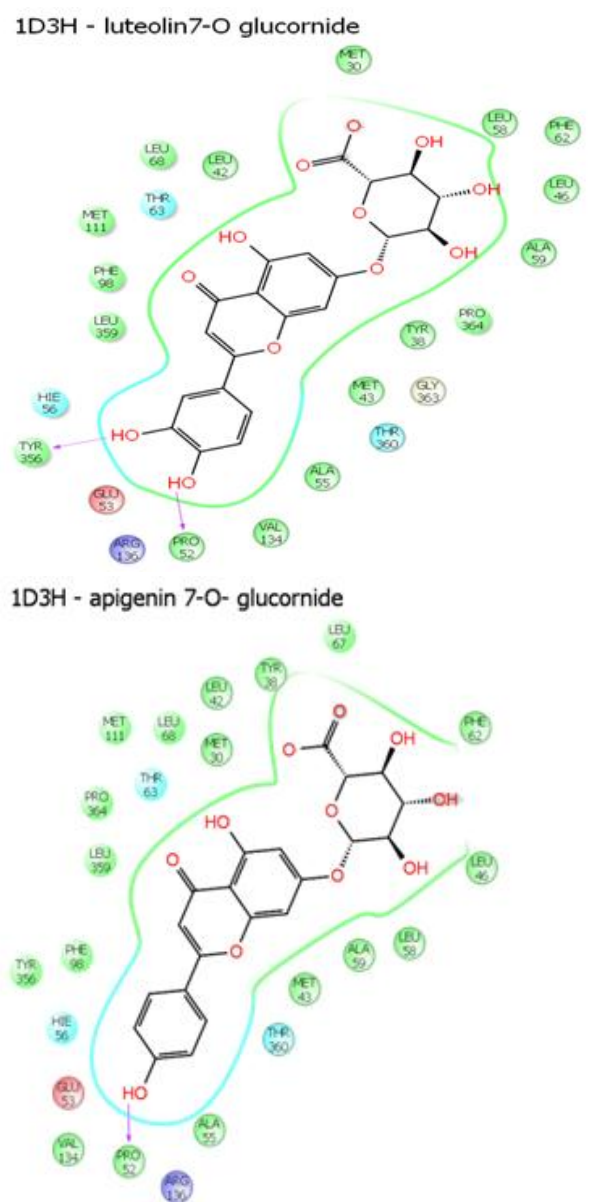

Fig. 7: Ligand Interaction Diagram for Luteolin 7O-glucornide and Apigenin 7O-glucornide.

Glide energy is the energy associated with binding of ligand with receptor. When the results were sorted according to Glide energy it was seen that Apigenin 7O- glucornide and Luteolin 7O- glucornide were the best hits. More negative energy indicates better binding. Thus the results suggest that compared to already known inhibitors the results obtained by docking with $C$. halicacabum phytomolecules were very significant. Figure 7 gives the Ligand Interaction Diagram description of Luteolin 7Oglucornide and Apigenin 7O-glucornide when docked with several known BindingDB inhibitors. It was seen that the two structures are chemically similar to each other except for a hydroxyl group missing in apigenin $7 \mathrm{O}$-glucornide. It was also observed that their binding mode is similar with hydrogen bonds at Pro52 of $\mathrm{hDHODH}$.

\section{Cross Docking}

Regular molecular docking considers the conformation of the target receptor to be rigid and the ligand to be flexible. This rigidity can account for the false positivity of the docking results. Thus a method called CROSS docking allows docking of all the different conformations of DHODH present in PDB with the 19 compounds. Cross docking tends to retrieve significant top hits with different conformations of the receptor to ultimately eliminate false positives. It's a class of flexible docking with receptor and ligand flexibility that justifies the docking poses of the ligand with different conformations of the receptor.

There were 22 different other receptors of DHODH for humans at the time of doing the experiment having a resolution ranging from $1.24 \AA$ to $3.00 \AA$ Á.

The Figure 8 gives a glimpse of all the 22 PDB structures of human DHODH superimposed on each other. The raspberry colored residue is ARG136 and cyan coloured residue is TYR356- part of the inhibitor binding site.

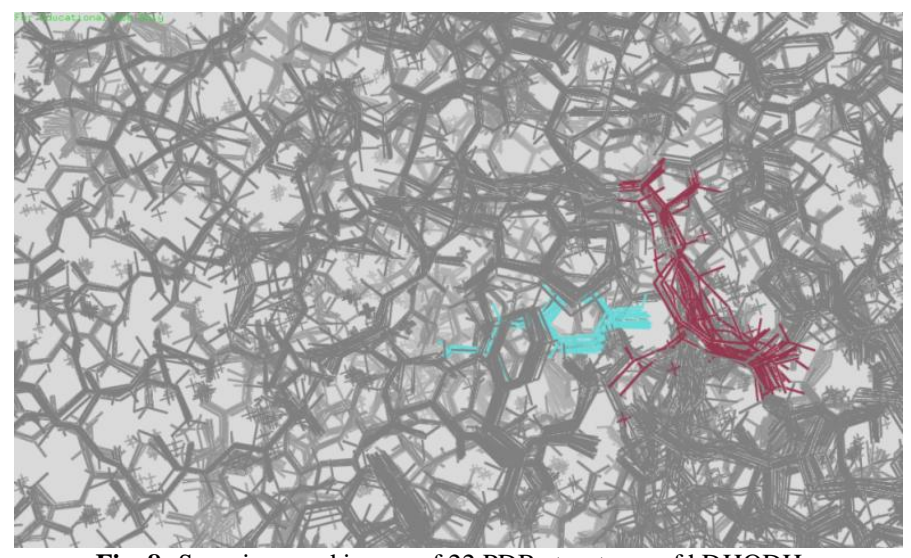

Fig. 8: Superimposed image of $22 \mathrm{PDB}$ structures of hDHODH

The cross docking results Table 6 depicts the best 3 hits of each receptor of human DHODH are displayed as the result table of cross docking. Luteolin 7O-glucornide had the highest XP G-score of $-15.913 \mathrm{Kcal} / \mathrm{mol}$ for the PDB structure 2BOM. For all the 22 cross docked results A771726 was lower than the top three hits. It was seen that all the resolutions from lowest to highest gave the same compounds as hits as from regular docking. Each hit was counted for the number of times it occurred as the first, second or the third hit for the different PDB id receptors. 
Table 6: Cross Docking score for 22 different DHODH based PDB Structures.

\begin{tabular}{|c|c|c|c|c|}
\hline PBD ID & Compound Name & 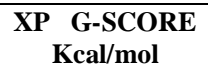 & $\begin{array}{c}\text { GLIDE ENERGY } \\
\text { Kcal/mol }\end{array}$ & Interactions \\
\hline \multirow[t]{3}{*}{$2 \mathrm{BOM}$} & Luteolin7O-glucornide & -15.978 & -57.419 & TYR356,ARG136,THR360 \\
\hline & Apigenin 70 -glucornide & -12.773 & -45.669 & PRO52,GLN47, ARG136,THR360 \\
\hline & Prunin & -12.624 & -62.943 & PRO52,ARG136,THR360 \\
\hline \multirow[t]{3}{*}{$2 \mathrm{PRH}$} & Luteolin 7O-glucornide & -15.821 & -66.246 & PHE62,LEU67, ARG136,THR360 \\
\hline & Prunin & -12.560 & -52.889 & TYR356,ARG136,VAL367 \\
\hline & Apigenin 7O-glucornide & -12.254 & -56.497 & TYR38,ARG136 \\
\hline \multirow{3}{*}{$1 \mathrm{D} 3 \mathrm{G}$} & Luteolin 7O-glucornide & -12.647 & -60.206 & TYR38,ARG136(2) \\
\hline & Prunin & -11.365 & -51.671 & ARG136,LEU(67) \\
\hline & Chlorogenic acid & -11.332 & -54.810 & PRO52,TYR38 \\
\hline \multirow[t]{3}{*}{$2 \mathrm{BXV}$} & Apigenin 7O-glucornide & -11.209 & -59.486 & MET30,LEU50,ARG136 \\
\hline & Chlorogenic acid & -10.766 & -54.689 & GLN47,TYR38,LEU67 \\
\hline & Luteolin 7O-glucornide & -10.645 & -54.488 & LEU42,ALA55 \\
\hline \multirow[t]{3}{*}{ 2PRL } & Luteolin 7O-glucornide & -14.804 & -64.290 & PRO52, LUE67,TYR356,ARG136 \\
\hline & Apigenin $7 \mathrm{O}$-glucornide & -13.595 & -46.383 & PRO52,GLN47,THR360,LEU359 \\
\hline & Prunin & -12.592 & -47.084 & PRO52,ARG136, \\
\hline \multirow[t]{3}{*}{$2 \mathrm{WV} 8$} & Luteolin 7 O-glucornide & -13.165 & -60.261 & PRO52, TYR356,ARG136 \\
\hline & Apigenin 7 O-glucornide & -12.543 & -52.731 & GLN47,PRO52,ARG136 \\
\hline & Prunin & -10.403 & -47.985 & PRO52,GLN47 \\
\hline \multirow[t]{3}{*}{ 2FPT } & Luteolin $7 \mathrm{O}$-glucornide & -12.908 & -51.039 & GLN47,ARG136 \\
\hline & Apigenin & -10.845 & -50.559 & ARG136(2) \\
\hline & Chlorogenic acid & -10.811 & -52.972 & ARG136,PRO52 \\
\hline \multirow[t]{3}{*}{$2 \mathrm{FPV}$} & Luteolin $7 \mathrm{O}$-glucornide & -13.666 & -66.036 & TYR356,ARG136,PRO52 \\
\hline & Prunin & -11.823 & -5.597 & ARG136,PRO52,TYR38 \\
\hline & Chlorogenic acid & -10.509 & -53.167 & ARG136,PRO52,TYR38 \\
\hline \multirow{3}{*}{$3 \mathrm{FJ} 6$} & Luteolin $7 \mathrm{O}$-glucornide & -13.883 & -55.947 & PRO52, LUE67,ARG136 \\
\hline & Apigenin $7 \mathrm{O}$-glucornide & -12.155 & -53.138 & PRO52,ARG136,THR360 \\
\hline & Chlorogenic acid & -11.066 & -57.702 & TYR38,PRO52,ARG136 \\
\hline \multirow[t]{3}{*}{$2 \mathrm{FPY}$} & Chlorogenic acid & -12.051 & -55.551 & TYR38,ARG136 \\
\hline & Luteolin $7 \mathrm{O}$-glucornide & -11.595 & -59.333 & TYR38,GLN47,ARG136 \\
\hline & Caftaric acid & -11.138 & -56.611 & LEU67,TYR360 \\
\hline \multirow[t]{3}{*}{ 2FQI } & Luteolin7 O-glucornide & -12.565 & -58.606 & TYR38,GLN47,ARG136 \\
\hline & Chlorogenic acid & -11.566 & -58.305 & TYR38,ARG136,GLN47,PRO52, \\
\hline & Caftaric acid & -11.472 & -56.901 & LEU67,TYR38,ARG136,GLN47,PRO52 \\
\hline \multirow{3}{*}{$3 \mathrm{~F} 1 \mathrm{Q}$} & Chlorogenic acid & -11.157 & -49.828 & PRO52 \\
\hline & Luteolin 7 O-glucornide & -10.290 & -50.543 & LEU42 \\
\hline & Phloridzin & -9.716 & -48.027 & PRO52 \\
\hline \multirow[t]{3}{*}{$3 \mathrm{FJL}$} & Luteolin 7 O-glucornide & -10.484 & -42.536 & PRO52,ARG136 \\
\hline & Phloridzin & -10.196 & -39.048 & LUE42,THR63,TYR356 \\
\hline & Apigenin $7 \mathrm{O}$-glucornide & -9.950 & -54.281 & PRO52,LEU42 \\
\hline $3 \mathrm{GOX}$ & Apigenin 7 O-glucornide & -12.469 & -53.739 & PRO52,ARG136(2),GLN47 \\
\hline & Luteolin 7 O-glucornide & -11.969 & -60.624 & ARG136,TYR38 \\
\hline & Prunin & -11.029 & -53.645 & GLN47,PRO52, ARG136 \\
\hline $3 \mathrm{GOU}$ & Luteolin 7 O-glucornide & -11.746 & -62.255 & PRO52,ARG136,GLN47,THR360 \\
\hline & Apigenin $7 \mathrm{O}$-glucornide & -10.095 & -55.482 & ARG136,TYR38 \\
\hline & Phloridzin & -9.601 & -47.349 & ARG136,TYR38 \\
\hline $3 \mathrm{U} 2 \mathrm{O}$ & Prunin & -12.715 & -54.631 & TYR356,PRO52(2),ARG136(2),THR360 \\
\hline & Luteolin 7 O-glucornide & -11.736 & -61.162 & ARG136(3),PRO52 \\
\hline & Apigenin $7 \mathrm{O}$-glucornide & -10.923 & -59.133 & LEU67,ARG136 \\
\hline $3 \mathrm{~W} 7 \mathrm{R}$ & Luteolin 7 O-glucornide & -14.443 & -65.248 & ARG136(2),PRO52,TYR356,LEU67 \\
\hline & Apigenin $7 \mathrm{O}$-glucornide & -14.078 & -59.445 & GLN47,ARG136(2) \\
\hline & Caftaric acid & -11.770 & -57.660 & LEU67(2),ARG136,THR360,PRO52 \\
\hline $3 Z W S$ & Caftaric acid & -10.614 & -54.308 & LEU67,TYR356,PRO52,THR63 \\
\hline & Chlorogenic acid & -10.612 & -46.071 & ARG136,GLN47 \\
\hline & Phloridzin & -9.249 & -42.920 & LEU67,HIS41(2) \\
\hline 4JGD & Phloridzin & -11.510 & -53.487 & TYR38,THR63,LEU359 \\
\hline & Luteolin 7 O-glucornide & -11.210 & -55.325 & ARG136,TYR38 \\
\hline & Apigenin $7 \mathrm{O}$-glucornide & --10.547 & -53.397 & LEU67,PRO52 \\
\hline 4JS3 & Luteolin 7 O-glucornide & -12.228 & -54.872 & LEU68(2),TYR38 \\
\hline & Prunin & -10.963 & -46.263 & TYR38,LEU68 \\
\hline & Apigenin 7 O-glucornide & -10.827 & -51.582 & PHE62,LEU68(2) \\
\hline 4JTT & Luteolin 7 O-glucornide & -11.446 & -53.933 & LEU68(2),PHE62 \\
\hline & Phloridzin & -10.682 & -45.420 & LEU359,PHE62,LEU68,TYR38 \\
\hline & Apigenin 7 O-glucornide & -10.639 & -49.430 & LEU68(2),TYR38 \\
\hline $4 \mathrm{JTU}$ & Luteolin 7 O-glucornide & -14.078 & -67.862 & ARG136(2),TYR356,PRO52 \\
\hline & Phloridzin & -11.460 & -48.299 & LEU67,ARG136 \\
\hline & Apigenin 7 O-glucornide & -11.013 & -58.578 & LEU67,ARG136 \\
\hline
\end{tabular}


The Table 7 below summarizes the cross docking results. It was seen that out of 22 cross docking results Luteolin $7 \mathrm{O}$ glucornide was obtained as the best hit majority of the time (15 times). This was followed by Apigenin 70- glucornide that was obtained as the second or the third hit majority of the times. These results indicate that Luteolin 7O- glucornide binds consistently with various conformations of human DHODH. The other compounds that were significantly bound to flexible hDHODH were prunin, Phloridzin, apigenin and caftaric acid. Then again caftaric acid did not bind in the same pocket as the other phytomolecules.

Table 7: Frequency of Hits in Cross Docking.

\begin{tabular}{cccc}
\hline COMPOUND & As 1st HIT & As 2nd HIT & As 3rd HIT \\
\hline Luteolin 7 O-Glucornide & 15 & 4 & 1 \\
Apigenin 7 O-Glucornide & 2 & 6 & 7 \\
Prunin & 1 & 3 & 4 \\
Phloridzin & 1 & 3 & 3 \\
Caftaric Acid & 2 & 2 & 3 \\
Chlorogenic Acid & 1 & 3 & 5 \\
Apigenin & 0 & 1 & 0 \\
\hline
\end{tabular}

Cross docking results emphasized on Luteolin 7Oglucornide and Apigenin 7O- glucornide to be significant along with all the previous validation methods used.

\section{MM/GBSA}

Any molecular docking experiment should ultimately be extrapolated to its dynamic behavior using molecular simulation. Thus the molecules to be simulated are decided upon by performing free binding energy calculations. MM/GBSA helps to hierarchically list the top best binding molecules of C. halicacabum. MM/GBSA predicted Apigenin 7O- glucornide followed by Luteolin 7O- glucornide as compounds having good binding energy compared to other phytocompounds of $\mathrm{C}$. halicacabum. Table 8 gives the Binding free energy and its individual contributions along with the results obtained in Glide $\mathrm{XP}$. The compounds are listed in the hierarchy as obtained from Glide XP. Only two compounds like Apigenin 7O-glucornide) and Luteolin 7O-glucornide $(-79.676 \mathrm{Kcal} / \mathrm{mol})$ showed better binding free energy than A771726 while caftaric acid had the lowest dG Bind score $(-35.346 \mathrm{Kcal} / \mathrm{mol})$. So out of the 6 top results obtained in Glide and previously employed methods only two compounds had significant dG Bind in Table 8. The lowest score is that of caftaric acid.

MM/GBSA results indicated that the contribution of Vanderwals (vdW) was higher in the top two protein ligand complexes (Apigenin 7O-glucornide and Luteolin 7O-glucornide) compared to the known ligand A771726 (Leflunomide). The vdW affinities were $(-60.24,-51.17$ and $-34.54 \mathrm{Kcal} / \mathrm{mol})$ from former to latter. On comparison of all the attributes predicted by MM/GBSA it was clearly noted that Lipophilic (Lipo) contribution is also a main component for binding affinities with Apigenin 7Oglucornide and Luteolin 7O-glucornide having lipophilic binding energy as -45.16 and $-46.98 \mathrm{Kcal} / \mathrm{mol}$ resp. compared to -31.02 $\mathrm{Kcal} / \mathrm{mol}$ for A771726.The other low scoring compounds like caftaric acid showed higher Columbic and solvation energies (145.9 and $-164.23 \mathrm{Kcal} / \mathrm{mol}$ respectively). These comparisons prove that good inhibitors show better vdW and Lipophilic contributions compared to non inhibitors. In other words better the lipophilic and Van der Waals interactions, better the binding energy of the inhibitor. Thus based on the MM/GBSA results Apigenin 7 O-glucornide and Luteolin 7O-glucornide were further taken for dynamics studies and compared with standard simulation of A771726 and Apo form of human DHODH protein.

Table 8: MM/GBSA results for binding free energy of ligand- DHODH receptor complex.

\begin{tabular}{|c|c|c|c|c|c|c|c|}
\hline 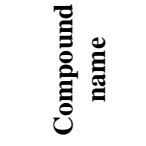 & & 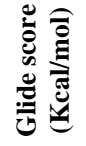 & 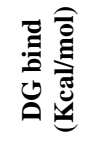 & 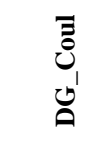 & $\frac{3}{3}$ & ڤ્. & $\begin{array}{l}\stackrel{0}{0} \\
\frac{\Delta}{\delta}\end{array}$ \\
\hline Caftaric Acid & & -11.286 & -35.346 & -145.929 & -32.969 & -21.167 & 164.233 \\
\hline $\begin{array}{l}\text { Luteolin } \\
\text { Glucornide }\end{array}$ & 7 & -10.842 & -79.676 & -39.353 & -51.612 & -46.986 & 50.549 \\
\hline Prunin & & -10.109 & -73.347 & -10.935 & -52.610 & -51.170 & 17.005 \\
\hline $\begin{array}{l}\text { Chlorogenic } \\
\text { acid }\end{array}$ & & -9.842 & -73.314 & -40.084 & -53.917 & -43.140 & 57.615 \\
\hline Chryseriol & & -9.186 & -67.408 & -11.885 & -38.950 & -35.922 & 13.274 \\
\hline $\begin{array}{l}\text { Apigenin } \\
\text { Glucornide }\end{array}$ & 7 & -8.213 & -80.659 & -31.600 & -60.246 & -45.162 & 46.341 \\
\hline A771726 & & -8.157 & -75.449 & -16.163 & -34.549 & -31.026 & 4.56 \\
\hline Cardiospermin & & -8.121 & -60.776 & -10.441 & -31.338 & -38.655 & 13.398 \\
\hline
\end{tabular}

\section{Molecular Dynamics}

Four complexes were taken for molecular simulation to evaluate their binding affinities with the human DHODH receptor. The apo form of hDHODH, hDHODH complexed with A771726, hDHODH complexed with Luteolin 7O-glucornide and hDHODH complexed with Apigenin 7O-glucornide was simulated for $10 \mathrm{~ns}$ and their RMSD was compared with that of A771726 and apo to check whether the hit compounds show a stable behavior with the receptor which is comparable with A771726 behavior and better than the apo receptor's behavior in a simulated environment of water molecules. The complexed proteins and their starting poses were taken from the cross docking files. Root mean square deviation [RMSD] is best means to measure the native behavior of a molecule in a simulation. Figure 9 shows the comparative plot of RMSD for the 4 receptor complexes. From the graph it was seen that among the 4 simulations, apo receptor showed a higher RMSD than known inhibitor, A771726 by $0.22 \AA$ while all the other simulations showed an RMSD closer to $1.5 \AA$. which is widely acceptable. This indicates that all the 4 simulations were stable after the 10ns MD simulation.

Table 9: Average RMSD of Receptor complexed with different ligands

\begin{tabular}{cc}
\hline hDHODH complexed with & Average RMSD \\
\hline Apo protein & 1.59 \\
Luteolin 7O-glucornide & 1.53 \\
Apigenin 7O-glucornide & 1.52 \\
A771726 & 1.51 \\
\hline
\end{tabular}

The lowest RMSD was exhibited by Luteolin 7Oglucornide complex at $1.46 \AA$ A. Table 9 gives the average RMSD values of the receptor with the ligand throughout 10ns MD 


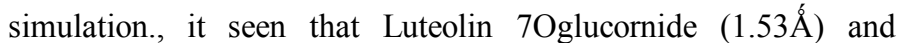
Apigenin $7 \mathrm{O}$-glucornide $(1.52 \AA \hat{)})$ are quite steady throughout the 10ns simulation from Table 9. Lower RMSD indicates good dynamics. To understand the stability factor in a better manner the number of $\mathrm{H}$-bonds formed between the hit compounds and receptor during 10ns simulation time was analyzed. The H-bonds analysis (Figure 10) showed that Luteolin 70-glucornide maintains on an average 2 hydrogen bond with residues like Tyr 356 and additionally interacts with Arg136, Ala55 throughout the whole simulation. Apigenin 7O-glucornide maintains one hydrogen bond with the inhibitor site residues, except from 4000ps to $6000 \mathrm{ps}$ where its forms additional $3 \mathrm{H}$ bonds on an average. In this period some water molecules come in closer contact with the inhibitor site thus leading to additional bonds. Apo was not analyzed for $\mathrm{H}$ bonds at the inhibitor site. The A771726 receptor complex started with $2 \mathrm{H}$ bonds initially but is initially begun with $2 \mathrm{H}$-bonds with Arg136 but all the $\mathrm{H}$ bond disappeared by the first 1000 ps itself which later re-establishes at 5000ps to form partially stable bond with Tyr147 which again disappears over time.
These H-bond interactions clearly indicate that the protein-ligand complexes of the hit compounds are stable and maintained with similar binding mode in the inhibitor site of human DHODH in the MD simulation studies. Figure 11 shows the RMSF plot of the residues present in the inhibitor binding site. The root mean square fluctuation was the least for Apigenin 70glucornide with $0.89 \AA \AA$ followed by $1.22 \AA$ for Luteolin 70 glucornide complex. Apo protein and A771726 complex receptor had $1.33 \AA$ fluctuations. At the $7^{\text {th }}$ ns simulation time all the complexes fluctuate to $6.5 \AA$ because of the water molecules intervention.

Figure 12 plots the ROG behavior of the simulated complexes. From the diagram it is quite clear that the radius of Gyration is predictably highest for apo DHODH $(20 \AA \hat{)})$ and the lowest for A771726 complex (16 ̊́). The hits compound complex had an average ROG value of $19 \AA$ at the end of 10 ns. From MD studies we infer that all hit compounds occupy the active site of human DHODH as known inhibitor, A771726 indicating that the hit compounds have similar bonded and non-bonded interactions.

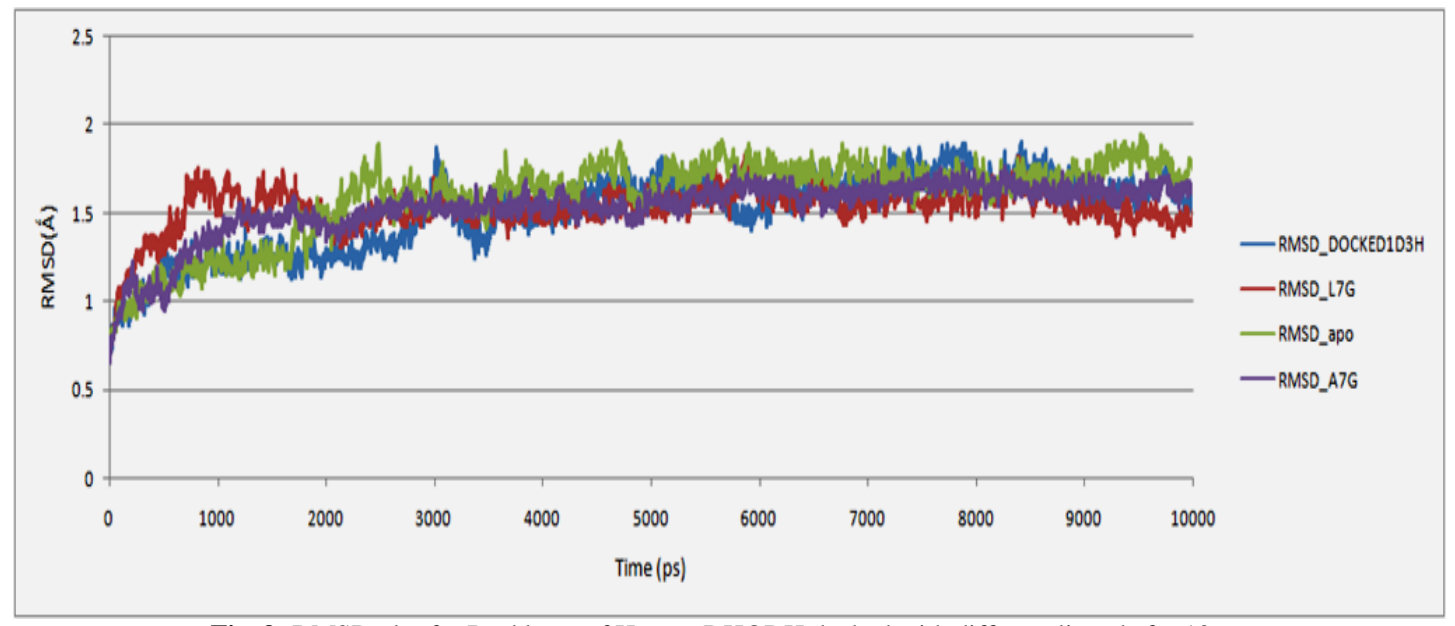

Fig. 9: RMSD plot for Backbone of Human DHODH docked with different ligands for 10ns.

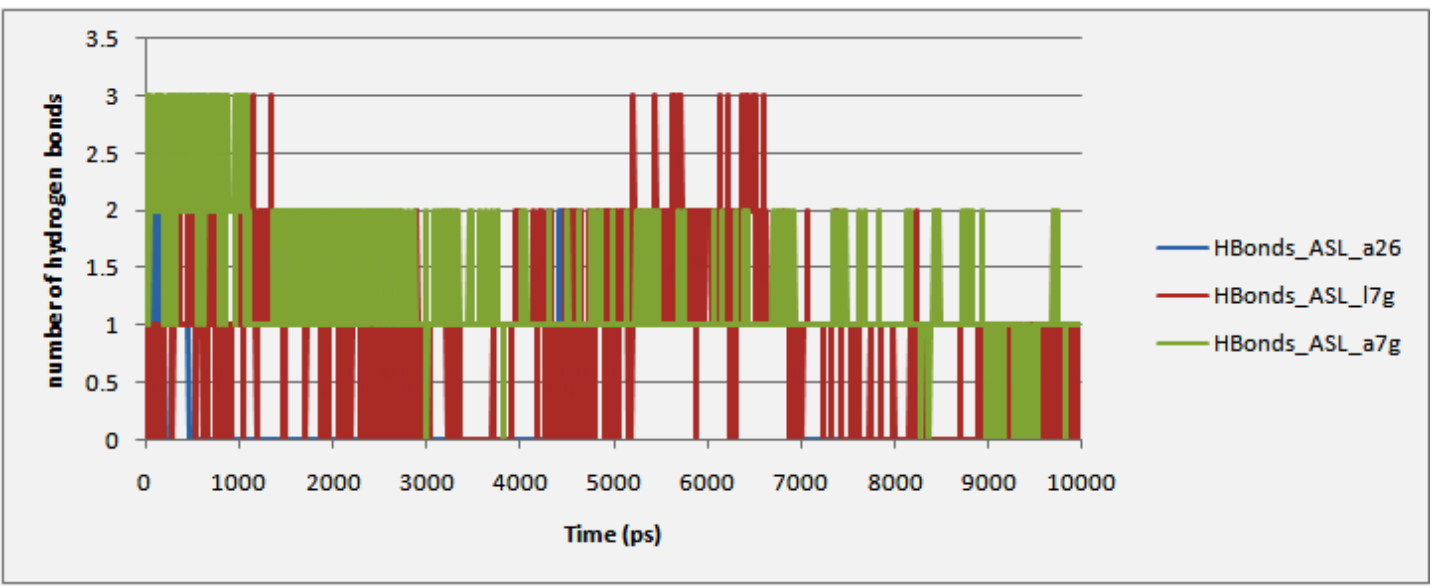

Fig. 10: Number of Hydrogen bond for (Arg136, Tyr356) of Human DHODH docked with different ligands for 10ns. 


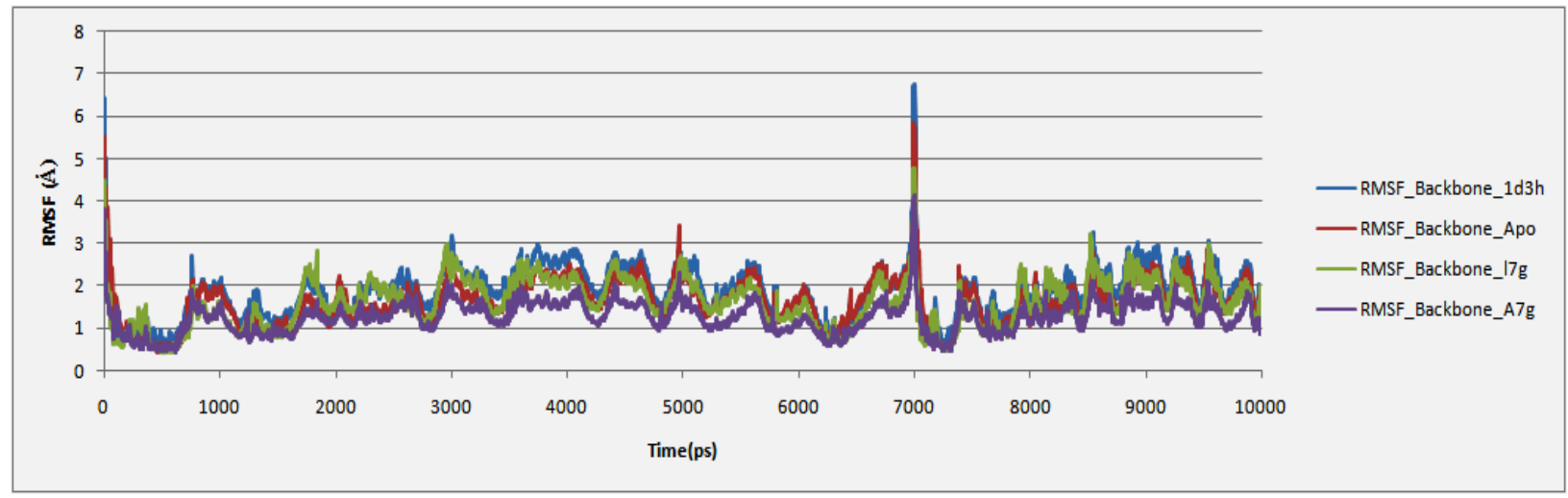

Fig. 11: RMSF for (Arg136, Tyr356) of hDHODH docked with different ligands for 10ns.

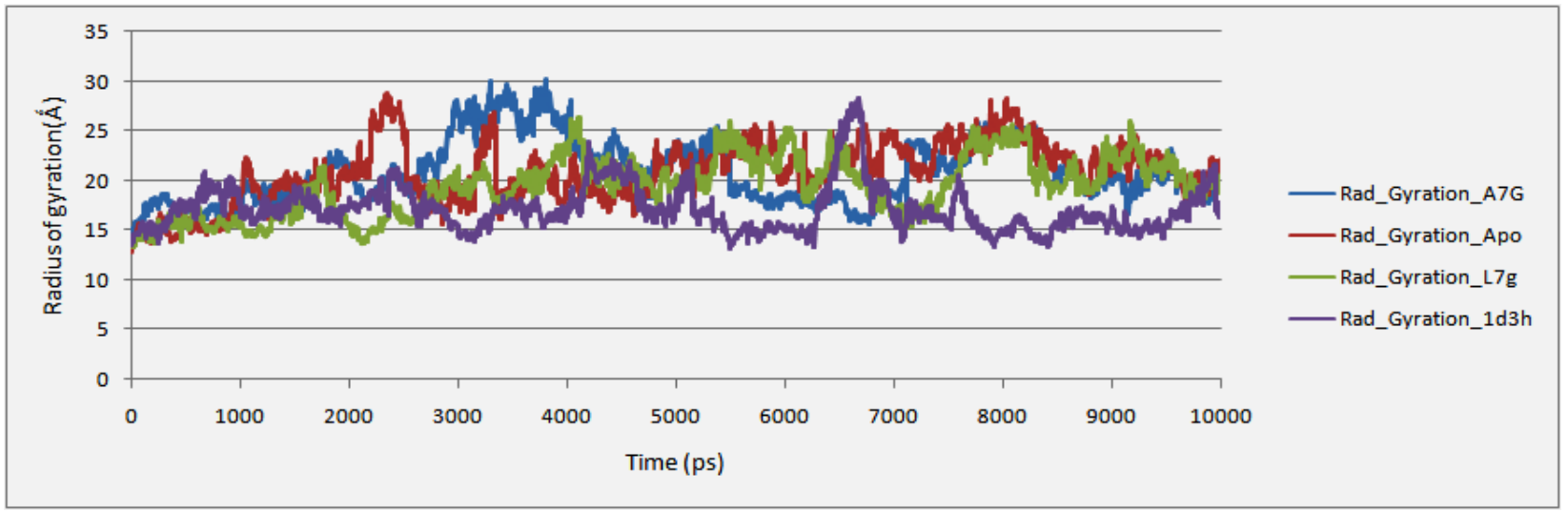

Fig. 12: ROG plot for Backbone of hDHODH docked with different ligands for 10ns.

\section{DISCUSSION}

C. halicacabum is a traditional medicinal plant used mainly for arthritis pain. It is widely known for its anti inflammatory properties in inflammatory diseases. The Leaf compounds were corroborated as influencing the interleukins and nitric oxide synthase to deplete the inflammation response (Sheeba and Asha, 2009). However, the target protein as well as the active compounds with anti-inflammatory profile is still unknown. Rheumatoid arthritis is an inflammatory disease where joints and cartilages progressively diminish and cause pain. Among the different targets that are widely used to reduce chronic inflammation, human DiHydroOrotate Dehydrogenase is widely studied. This target is inhibited by a special class of drugs called Disease Modifying Anti Rheumatic Drug [DMARD]. Currently, many studies are performed to seek out valid alternatives to the drugs available that are practically amenable for long term usage. This precisely is the driving force behind our study. The phytocompounds of $C$. halicacabum were screened, docked and simulated against hDHODH to evaluate the possible inhibitory effects of these compounds on hDHODH.

The 19 compounds in the leaf of $C$. halicacabum were used to screen a validated $\mathrm{E}$ pharmacophore specific for an inhibitor of hDHODH. The 5 hits obtained though not of a very high fitness score indicate that desirable features of a good inhibitor of hDHODH are present in the phytocompounds of $C$. halicacabum. Further the phytocompounds were docked with the receptor of $\mathrm{hDHODH}$ to obtain 9 hits having a XP Gscore better than the known inhibitor of hDHODH - A771726. The top hit caftaric acid interacted in a pocket deeper than the known inhibitor site. Caftaric acid although gave very high score of $-11.56 \mathrm{Kcal} /$ mol, bound to a FMN binding site rather than the A771726 binding site. This could have serious repercussions as hDHODH is a 2 site catalytic enzyme for both orotate as well as ubiquinone reduction. Thus the binding of caftaric acid to a different site can cross react with oxidative phosphorylation process. The active site residues Arg136 and Tyr356 and the surrounding $3 \AA$ amino acids were the main binding site for all the other hits mainly apigenin 7O-glucornide and Luteolin 7O-glucornide, chlorogenic acid and pruning. The compounds obtained were checked for its credibility by applying additional validation tests like varying docking algorithms, docking with decoys and with other known inhibitors of hDHODH. Further a flexible ligand receptor docking protocol was performed using crossdocking of other known crystal structures of $\mathrm{hDHODH}$ to find a lead ligand and its conformational pose.

Thus multiple cross dockings showed majority of the times Luteolin 7O-glucornide as the best hit followed by Apigenin 7O-glucornide. The poses of the cross docked receptor along with the ligand were taken as the starting structure for dynamics. The 
simulation time of $10 \mathrm{~ns}$ was used so as to get an initial preview on the type of interactions and the forces that act against the receptor ligand interactions. Among four complexes simulated, the apo protein had a high RMSD value of $1.59 \AA$ compared to the other 3 complexes. The lead compounds Luteolin 7O-glucornide and Apigenin 7O- glucornide complexed receptor were comparable and equally good as the known inhibitor A771726 (1.53, 1.52. $1.51 \AA$ resp.) Similar study of this kind has been used to find inhibitors to benign prostatic hyperplasia (Wang et al., 2014) and Low Density Lipoprotein formation (Jiang et al., 2016).Our results indicate that the leaf compounds Luteolin 7O-glucornide and Apigenin 7O- glucornide are the principal ingredients of $C$. halicacabum that alleviate that disease of Rheumatoid arthritis by acting on the pyrimidine pathway target of hDHODH. Further, enzyme inhibition studies are warranted.

\section{CONCLUSION}

A study to find novel inhibitors for hDHODH was done using E- pharmacophore screening, cross docking and molecular dynamics. All the validations and simulations performed on the complex of hDHODH with the lead-like compounds Luteolin 7Oglucornide and Apigenin 7O-glucornide resulted in giving a broad idea on the type of interactions that may occur between $C$. halicacabum leaf compounds and the target hDHODH. This study also provides a foundation for efficiency studies of $C$. halicacabum against a target of Rheumatoid arthritis. The study helps to spell out active ingredients in $C$. halicacabum leaf that may further be used for treatment of Rheumatoid arthritis. Thus Luteolin 7O-glucornide and Apigenin 7O-glucornide can be shortlisted as lead compounds which can be optimized for stability. Alternately, our study can be used as a starting point for exploring an alternate mechanism of action of the leaves of $C$. halicacabum against inflammatory targets like hDHODH.

\section{Acknowledgement}

We the authors acknowledge SRM University and the facilities provided by the college for the successful completion of work.

\section{Financial support and sponsorship: Nil.}

Conflict of Interests: There are no conflicts of interest.

\section{REFERENCES}

Babu K and Krishnakumari S. Anti-inflammatory and antioxidant compound, rutin in Cardiospermum halicacabum leaves. Anc Sci Life, 2005; 25: 47.

DeLeve L and Kaplowitz N. 2013. Drug-induced liver disease. Academic, Oxford.

Du J, Sun H, Xi L, Li J, Yang Y and Liu H et al. Molecular modeling study of checkpoint kinase 1 inhibitors by multiple docking strategies and prime/MM-GBSA calculation. J Comp Chem, 2011; 32: 2800-2809.

Eldridge M, Murray C, Auton T, Paolini G and Mee R. Empirical scoring functions: I. The development of a fast empirical scoring function to estimate the binding affinity of ligands in receptor complexes. J Comput-Aided Mol Des, 1997;11: 425-445.
Fox R, Herrmann M, Frangou C, Wahl G, Morris R and Strand $\mathrm{V}$ et al. Mechanism of Action for Leflunomide in Rheumatoid Arthritis. J Clin Immunol, 1999; 93: 198-208.

Fritzson I, Svensson B, Al-Karadaghi S, Walse B, Wellmar U and Nilsson U. et al.. Inhibition of Human DHODH by 4Hydroxycoumarins, Fenamic Acids, andN-(Alkylcarbonyl)anthranilic Acids Identified by Structure-Guided Fragment Selection. ChemMedChem, 2010; 5: 608-617.

Ganesan K, Sehgal P, Mandal A and Sayeed S. Protective Effect of Withania somnifera and Cardiospermum halicacabum Extracts Against Collagenolytic Degradation of Collagen. Appl Biochem Biotech, 2011; 165: 1075-1091.

Govindarajan M and Sivakumar R. Repellent properties of Cardiospermum halicacabum Linn. (Family: Sapindaceae) plant leaf extracts against three important vector mosquitoes. Asian Pac J Trop Biomed, 2012; 2: 602-607.

Hsu K, Chen Y, Lin S and Yang J. iGEMDOCK: a graphical environment of enhancing GEMDOCK using pharmacological interactions and post-screening analysis. BMC Bioinformatics, 2011;12: S33.

Huang M, Huang S, Wang B, Wu C, Sheu M and Hou W. et al. Antioxidant and anti-inflammatory properties of Cardiospermum halicacabum and its reference compounds ex vivo and in vivo. J Ethnopharmacol, 2011; 133: 743-750.

Jeyadevi R, Sivasudha T, Ilavarasi A and Thajuddin N. Chemical Constituents and Antimicrobial Activity of Indian Green Leafy Vegetable Cardiospermum halicacabum. Indian J Microbiol, 2012; 53: 208-213.

Jeyadevi R, Sivasudha T, Rameshkumar A and Dinesh Kumar L. Anti-arthritic activity of the Indian leafy vegetable Cardiospermum halicacabum in Wistar rats and UPLC-QTOF-MS/MS identification of the putative active phenolic components. Inflamm Res, 2012; 62: 115-126.

Jiang L, He Y, Luo G, Yang Y, Li G and Zhang Y. Discovery of potential novel microsomal triglyceride transfer protein inhibitors via virtual screening of pharmacophore modelling and molecular docking. Mol Simul, 2016; 42: 1223-1232.

Jorgensen W. The Many Roles of Computation in Drug Discovery. Science, 2004; 303: 1813-1818.

Karplus M and McCammon J. Molecular dynamics simulations of biomolecules. Nat Struct Biol, 2002; 9: 646-652.

Kirchmair J, Göller A, Lang D, Kunze J, Testa B and Wilson I et al. Predicting drug metabolism: experiment and/or computation?. Nat Rev Drug Discov, 2015; 14: 387-404.

Madhavi Sastry G, Adzhigirey M, Day T, Annabhimoju R and Sherman W. Protein and ligand preparation: parameters, protocols, and influence on virtual screening enrichments. J Comput-Aided Mol Des, 2013; 27: 221-234.

Marrone T, Briggs J and McCammon J. Structure-Based Drug Design:Computational Advances. Annu Rev Pharmacol Toxicol, 1997; 37 71-90.

Munier-Lehmann H, Vidalain P, Tangy $\mathrm{F}$ and Janin Y. On Dihydroorotate Dehydrogenases and Their Inhibitors and Uses. J Med Chem, 2013; 56: 3148-3167.

Nicola G, Liu T, Hwang L and Gilson M. BindingDB: A Protein-Ligand Database for Drug Discovery. Biophys J, 2012; 102: 61a.

Pillai N and Santhakumari G. Anti-Arthritic and AntiInflammatory Actions of Nimbidin. Planta Med, 1981; 43: 59-63.

Qi Q, Lu X, Li Y, Fan Y and Zhu J. F01WB-1315 A and B, two dihydroorotate dehydrogenase inhibitors from microbial metabolites. Wei sheng wu xue bao= Acta microbiologica Sinica, 2009; 49: 485-491.

Raza A. Review of beneficial and remedial aspects of Cardiospermum halicacabum L. Afr J Pharm Pharmacol, 2013; 7: 30263033.

Sadique J, Chandra T, Thenmozhi V and Elango V. Biochemical modes of action of Cassia occidentalis and Cardiospermum halicacabum in inflammation. J Ethnopharmacol, 1987; 19: 201-212.

Sheeba M and Asha V. Cardiospermum halicacabum ethanol extract inhibits LPS induced COX-2, TNF- $\alpha$ and iNOS expression, which is mediated by NF- $\mathrm{kB}$ regulation, in RAW264.7 cells. J Ethnopharmacol, 2009; 124: 39-44. 
Sotriffer C. Virtual screening, 1st ed. Wiley-VCH, 2011; Weinheim, Germany.

Swaminathan P, Kalva S and Saleena L. E-Pharmacophore and Molecular Dynamics Study of Flavonols and Dihydroflavonols as Inhibitors Against DiHydroOrotate DeHydrogenase. Comb Chem High Throughput Screen, 2014; 17: 663-673.

Totrov $M$ and Abagyan R. Flexible ligand docking to multiple receptor conformations: a practical alternative. Curr Opin Struct Biol, 2008; 18: 178-184.

Triballeau N, Acher F, Brabet I, Pin J and Bertrand H. Virtual Screening Workflow Development Guided by the "Receiver Operating Characteristic" Curve Approach. Application to High-Throughput Docking on Metabotropic Glutamate Receptor Subtype 4. J Med Chem, 2005; 48: 2534-2547.

Veeramani C, Al-Numair K, Alsaif M, Chandramohan G, AlNumair $\mathrm{N}$ and Pugalendi K. Protective effect of Cardiospermum halicacabum leaf extract on glycoprotein components on STZ-induced hyperglycemic rats. Asian Pac J Trop Med, 2012; 5: 939-944.

Venkatachalam C, Jiang, X, Oldfield T, and Waldman $\mathrm{M}$. LigandFit: a novel method for the shape-directed rapid docking of ligands to protein active sites. J Mol Graph Model, 2003; 21: 289-307.
Venkatesh B and Krishnakumari S. Cardiospermum halicacabum suppresses the production of TNF-alpha and nitric oxide by human peripheral blood mononuclear cells. Afr J Biomed Res, 2009; 9.

Vyas V. Virtual Screening: A Fast Tool for Drug Design. Sci Pharm, 2008; 76: 333-360.

Wang J, Liu H, Zhou Z, Chen W and Ho Y. Discovery of novel $5 \alpha$-reductase type II inhibitors by pharmacophore modelling, virtual screening, molecular docking and molecular dynamics simulations. Mol Simul, 2014; 41: 287-297.

Zalke AS, Duraiswamy B, Gandagule UB, Singh N. Pharmacognostical evaluation of Cardiospermum halicacabum Linn. Anc Sci Life. 20131; 33(1):15-21

\section{How to cite this article:}

Swaminathan P., Saleena L. Evaluation of Cardiospermum halicacabum leaf compounds against human DihydroOrotate Dehydrogenase- a target for Rheumatoid Arthritis using Structure based Drug Designing. J App Pharm Sci, 2017 ; 7 (08): 048-061. 